\title{
Erratum to: Multivariate McCormick relaxations
}

\author{
Jaromił Najman $^{1}$ - Dominik Bongartz ${ }^{1} \cdot$ Angelos Tsoukalas $^{2}$ • \\ Alexander Mitsos ${ }^{1}$
}

Published online: 25 October 2016

(C) Springer Science+Business Media New York 2016

\section{Erratum to: J Glob Optim (2014) 59:633-662 DOI 10.1007/s10898-014-0176-0}

\begin{abstract}
We provide a correction of the closed-form solutions for the multivariate McCormick relaxations of the binary product provided by Tsoukalas and Mitsos (JOGO, 59:633-662, 2014). The original closed-form solution may provide a function that is a nonconvex relaxation or a convex function that is not a relaxation or a function that is neither convex nor a valid relaxation in some special cases. We prove the validity of the new closedform solution.
\end{abstract}

In [1] Tsoukalas and Mitsos introduced the multivariate McCormick relaxations and in particular the multivariate McCormick relaxation of the binary product of functions. To provide a better overview, in the following we only consider the convex relaxation in detail. All results are analogously applicable to the concave relaxation for which we directly provide the closed-form solution. We adopt all assumptions made in [1]. The convex relaxation of $g(z)=\operatorname{mult}\left(f_{1}(z), f_{2}(z)\right) \equiv f_{1}(z) f_{2}(z)$ with $f_{i}: Z \subset \mathbb{R}^{n} \rightarrow \mathbb{R}$ is given by:

$$
\begin{array}{r}
g^{c v}(\boldsymbol{z})=\min _{x_{i} \in\left[f_{i}^{L}, f_{i}^{U}\right]} \max \left\{H_{1}(\boldsymbol{x}), H_{2}(\boldsymbol{x})\right\} \\
\text { s.t. } f_{1}^{c v}(\boldsymbol{z}) \leq x_{1} \leq f_{1}^{c c}(\boldsymbol{z}) \\
f_{2}^{c v}(\boldsymbol{z}) \leq x_{2} \leq f_{2}^{c c}(\boldsymbol{z})
\end{array}
$$

The online version of the original article can be found under doi:10.1007/s10898-014-0176-0.

$凶$ Alexander Mitsos

amitsos@alum.mit.edu

1 RWTH Aachen University, AVT - Aachener Verfahrenstechnik, Process Systems Engineering, Turmstrasse 46, 52056 Aachen, Germany

2 Olayan School of Business, American University of Beirut, Riad El-Solh Beirut 1107 2020, Lebanon 
with

$$
H_{1}(\boldsymbol{x})=f_{2}^{L} x_{1}+f_{1}^{L} x_{2}-f_{1}^{L} f_{2}^{L}, H_{2}(\boldsymbol{x})=f_{2}^{U} x_{1}+f_{1}^{U} x_{2}-f_{1}^{U} f_{2}^{U} .
$$

The concave relaxation is given by:

$$
\begin{gathered}
g^{c c}(z)=\max _{x_{i} \in\left[f_{i}^{L}, f_{i}^{U}\right]} \min \left\{f_{2}^{L} x_{1}+f_{1}^{U} x_{2}-f_{1}^{U} f_{2}^{L}, f_{2}^{U} x_{1}+f_{1}^{L} x_{2}-f_{1}^{L} f_{2}^{U}\right\} \\
\text { s.t. } f_{1}^{c v}(z) \leq x_{1} \leq f_{1}^{c c}(z) \\
f_{2}^{c v}(z) \leq x_{2} \leq f_{2}^{c c}(z)
\end{gathered}
$$

where $f_{i}^{L}, f_{i}^{U}$ denote bounds for $f_{i}$, i.e., $f_{i}^{L} \leq f_{i}(z) \leq f_{i}^{U}$ for all $z \in Z$ and $f_{i}^{c v}, f_{i}^{c c}$ are convex and concave relaxations of $f_{i}$.

In [1] the authors also provide a closed-form solution for relaxation (1). The closed form they provide for the convex relaxation is given by

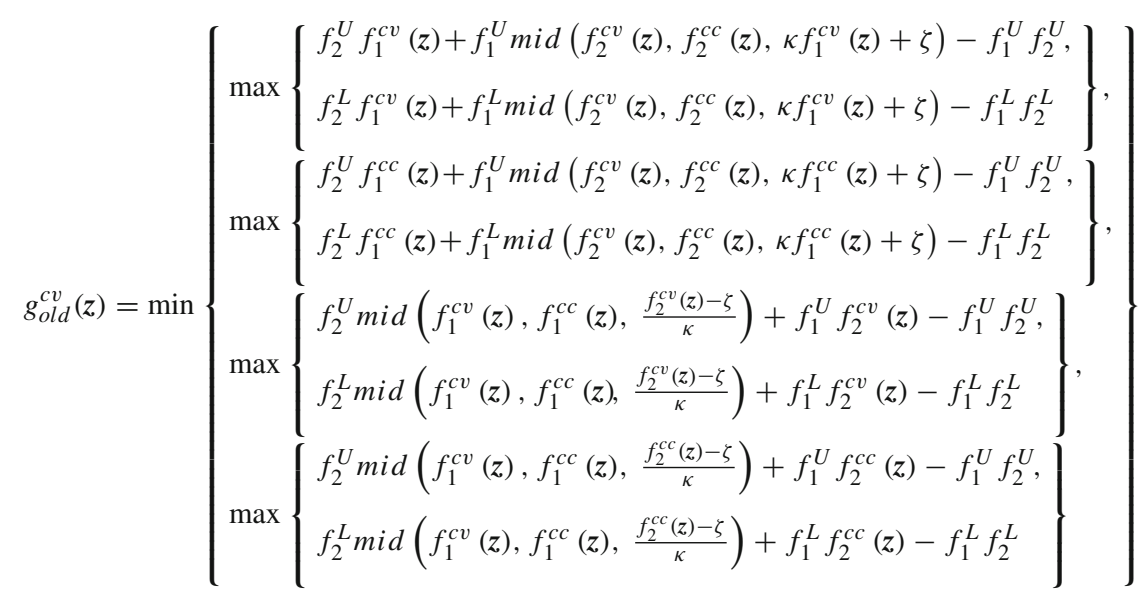

with

$$
\kappa=\frac{f_{2}^{L}-f_{2}^{U}}{f_{1}^{U}-f_{1}^{L}}, \zeta=\frac{f_{1}^{U} f_{2}^{U}-f_{1}^{L} f_{2}^{L}}{f_{1}^{U}-f_{1}^{L}} .
$$

In the following, we show that the closed-form solution (3) is not always correct. More specifically, in some special cases it is neither convex nor a valid relaxation, see Fig. 1, and sometimes it is convex but not a relaxation, see Fig. 2. We give a simple counter example for each case and discuss the issue. Subsequently, we provide a corrected closed-form solution for the multivariate McCormick binary product of functions.

Example 1 Consider $g(z)=\operatorname{mult}\left(f_{1}(z), f_{2}(z)\right)$ with $f_{1}(z)=(z+1)^{2}$ and $f_{2}(z)$ $=(z-1)^{6}+1$ on $Z=[0,1]$. We use exact bounds for $f_{1}, f_{2}$ given by $f_{1}^{L}=1, f_{1}^{U}=4$, $f_{2}^{L}=1, f_{2}^{U}=2$. We use envelopes for the relaxations of $f_{1}, f_{2}$ given by:

$$
\begin{array}{ll}
f_{1}^{c v}(z)=(z+1)^{2}, & f_{1}^{c c}(z)=1+3 z \\
f_{2}^{c v}(z)=(z-1)^{6}+1, & f_{2}^{c c}(z)=2-z .
\end{array}
$$



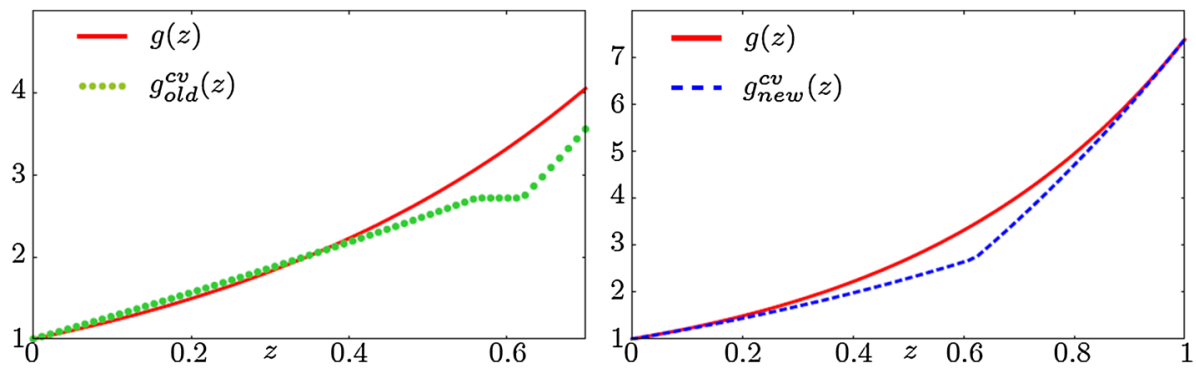

Fig. 1 The old wrong closed form $g_{\text {old }}^{c v}(3)$ gives a function that is neither convex nor a valid relaxation. The new correct formula $g_{\text {new }}^{c v}(5)$ provides the desired convex relaxation for $g(z)=\exp (z) \cdot \exp (z)$ on $Z=[0,1]$. Note that $g_{\text {old }}^{c v}$ is plotted only over $Z=[0,0.7]$ to make the issues more visible

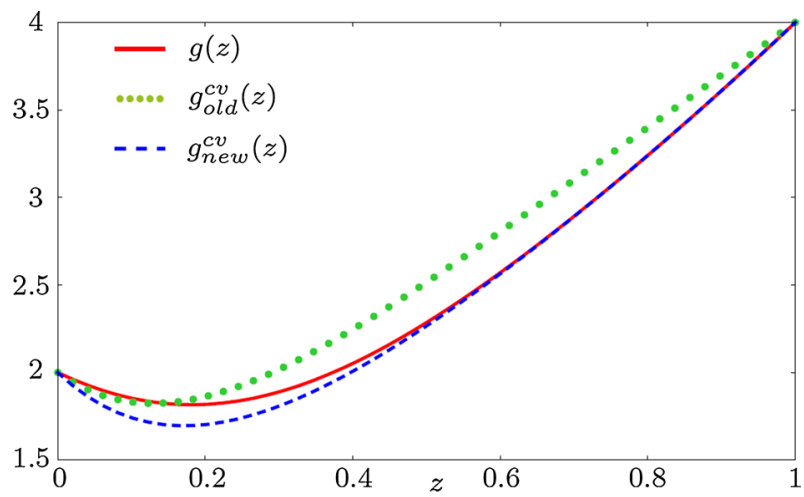

Fig. 2 The old wrong closed form $g_{\text {old }}^{c v}$ (3) gives a convex but not valid relaxation, while the new correct formula $g_{\text {new }}^{c v}(5)$ provides the convex relaxation for $g(z)=(z+1)^{2}\left((z-1)^{6}+1\right)$ on $Z=[0,1]$

When we evaluate (3), we get $\kappa=-\frac{1}{3}, \zeta=\frac{7}{3}$ and see that $g_{\text {old }}^{c v}(z)$ is given by

$$
\begin{aligned}
g_{\text {old }}^{c v}(z) & =\max \left\{\begin{array}{c}
f_{2}^{U} \operatorname{mid}\left(f_{1}^{c v}(z), f_{1}^{c c}(z), \frac{f_{2}^{c v}(z)-\zeta}{\kappa}\right)+f_{1}^{U} f_{2}^{c v}(z)-f_{1}^{U} f_{2}^{U}, \\
f_{2}^{L} \operatorname{mid}\left(f_{1}^{c v}(z), f_{1}^{c c}(z), \frac{f_{2}^{c v}(z)-\zeta}{\kappa}\right)+f_{1}^{L} f_{2}^{c v}(z)-f_{1}^{L} f_{2}^{L}
\end{array}\right\} \\
& =\max \left\{\begin{array}{c}
2 \operatorname{mid}\left(f_{1}^{c v}(z), f_{1}^{c c}(z), 7-3 f_{2}^{c v}(z)\right)+4 f_{2}^{c v}(z)-8, \\
\operatorname{mid}\left(f_{1}^{c v}(z), f_{1}^{c c}(z), 7-3 f_{2}^{c v}(z)\right)+f_{2}^{c v}(z)-1
\end{array}\right\} \\
& =\operatorname{mid}\left(f_{1}^{c v}(z), f_{1}^{c c}(z), 7-3 f_{2}^{c v}(z)\right)+f_{2}^{c v}(z)-1 \\
& =f_{1}^{c c}(z)+f_{2}^{c v}(z)-1 \\
& =(z-1)^{6}+3 z+1
\end{aligned}
$$

As can be seen in Fig. 2, the resulting function $g_{\text {old }}^{c v}$ is convex but not a relaxation of $g$.

We now discuss what causes the mistake and give a correct closed-form solution for (1). The envelope of the binary product in Example 1 is constructed over the exact bounds of $f_{1}, f_{2}$ given by $X=\left[f_{1}^{L}, f_{1}^{U}\right] \times\left[f_{2}^{L}, f_{2}^{U}\right]$ and is strictly monotonically increasing over $X$ with its minimum in the corner point $\left(f_{1}^{L}, f_{1}^{U}\right)$. The minimum of the envelope over the box given by $\left[f_{1}^{c v}(z), f_{1}^{c c}(z)\right] \times\left[f_{2}^{c v}(z), f_{2}^{c c}(z)\right]$ is then attained in the corner point $\left(f_{1}^{c v}(z), f_{2}^{c v}(z)\right)$. 
Formula (3) falsely gives the corner point $\left(f_{1}^{c c}(z), f_{2}^{c v}(z)\right)$ as the solution of formulation (1) because it holds that

$$
\operatorname{mid}\left(f_{1}^{c v}(z), f_{1}^{c c}(z), \frac{f_{2}^{c v}(z)-\zeta}{\kappa}\right)=f_{1}^{c c}(z) \text { over } Z=[0,1] .
$$

Note that similar examples can be constructed where (3) excludes the optimal corner $\left(f_{1}^{c c}(z), f_{2}^{c c}(z)\right)$ for the convex relaxation and the corners $\left(f_{1}^{c v}(z), f_{2}^{c c}(z)\right), \quad\left(f_{1}^{c c}(z)\right.$, $\left.f_{2}^{c v}(z)\right)$ for the concave relaxation. In the proof of Lemma 1, it becomes clear why the corners $\left(f_{1}^{c v}(\boldsymbol{z}), f_{2}^{c c}(\boldsymbol{z})\right),\left(f_{1}^{c c}(\boldsymbol{z}), f_{2}^{c v}(\boldsymbol{z})\right)$ cannot be excluded by the mid (..) term when computing the convex relaxation. The same argumentation applies to the corners $\left(f_{1}^{c v}(z), f_{2}^{c v}(z)\right),\left(f_{1}^{c c}(z), f_{2}^{c c}(z)\right)$ when regarding the concave relaxation.

To avoid the exclusions described above, we correct the current closed-form solutions by explicitly adding the two corners that can be excluded by the mid (...) terms in the case of a monotonic envelope of the binary product. We add $\left(f_{1}^{c v}(\boldsymbol{z}), f_{2}^{c v}(\boldsymbol{z})\right),\left(f_{1}^{c c}(\boldsymbol{z}), f_{2}^{c c}(\boldsymbol{z})\right)$ to the closed form of the multivariate convex relaxation and we add the corners $\left(f_{1}^{c v}(z), f_{2}^{c c}(z)\right),\left(f_{1}^{c c}(z), f_{2}^{c v}(z)\right)$ to the closed form of the multivariate concave relaxation. The new closed-form solutions for the multivariate McCormick relaxations of the binary product of functions are then given by

$$
g_{\text {new }}^{c v}(z)=\min \left\{\begin{array}{l}
\max \left\{\begin{array}{l}
f_{2}^{U} f_{1}^{c v}(z)+f_{1}^{U} \operatorname{mid}\left(f_{2}^{c v}(z), f_{2}^{c c}(z), \kappa f_{1}^{c v}(z)+\zeta\right)-f_{1}^{U} f_{2}^{U}, \\
f_{2}^{L} f_{1}^{c v}(z)+f_{1}^{L} \operatorname{mid}\left(f_{2}^{c v}(z), f_{2}^{c c}(z), \kappa f_{1}^{c v}(z)+\zeta\right)-f_{1}^{L} f_{2}^{L}
\end{array}\right\}, \\
\max \left\{\begin{array}{l}
f_{2}^{U} f_{1}^{c c}(z)+f_{1}^{U} \operatorname{mid}\left(f_{2}^{c v}(z), f_{2}^{c c}(z), \kappa f_{1}^{c c}(z)+\zeta\right)-f_{1}^{U} f_{2}^{U}, \\
f_{2}^{L} f_{1}^{c c}(z)+f_{1}^{L} \operatorname{mid}\left(f_{2}^{c v}(z), f_{2}^{c c}(z), \kappa f_{1}^{c c}(z)+\zeta\right)-f_{1}^{L} f_{2}^{L}
\end{array}\right\}, \\
\max \left\{\begin{array}{l}
f_{2}^{U} \operatorname{mid}\left(f_{1}^{c v}(z), f_{1}^{c c}(z), \frac{f_{2}^{c v}(z)-\zeta}{\kappa}\right)+f_{1}^{U} f_{2}^{c v}(z)-f_{1}^{U} f_{2}^{U}, \\
f_{2}^{L} \operatorname{mid}\left(f_{1}^{c v}(z), f_{1}^{c c}(z), \frac{f_{2}^{c v}(z)-\zeta}{\kappa}\right)+f_{1}^{L} f_{2}^{c v}(z)-f_{1}^{L} f_{2}^{L}
\end{array}\right\}, \\
\max \left\{\begin{array}{l}
f_{2}^{U} \operatorname{mid}\left(f_{1}^{c v}(z), f_{1}^{c c}(z), \frac{f_{2}^{c c}(z)-\zeta}{\kappa}\right)+f_{1}^{U} f_{2}^{c c}(z)-f_{1}^{U} f_{2}^{U}, \\
f_{2}^{L} \operatorname{mid}\left(f_{1}^{c v}(z), f_{1}^{c c}(z), \frac{f_{2}^{c c}(z)-\zeta}{\kappa}\right)+f_{1}^{L} f_{2}^{c c}(z)-f_{1}^{L} f_{2}^{L}
\end{array}\right\}, \\
\max \left\{\begin{array}{l}
f_{2}^{U} f_{1}^{c v}(z)+f_{1}^{U} f_{2}^{c v}(z)-f_{1}^{U} f_{2}^{U}, \\
f_{2}^{L} f_{1}^{c v}(z)+f_{1}^{L} f_{2}^{c v}(z)-f_{1}^{L} f_{2}^{L}
\end{array}\right\}, \\
\max \left\{\begin{array}{l}
f_{2}^{U} f_{1}^{c c}(z)+f_{1}^{U} f_{2}^{c c}(z)-f_{1}^{U} f_{2}^{U}, \\
f_{2}^{L} f_{1}^{c c}(z)+f_{1}^{L} f_{2}^{c c}(z)-f_{1}^{L} f_{2}^{L}
\end{array}\right\}
\end{array}\right\}
$$

with

$$
\kappa=\frac{f_{2}^{L}-f_{2}^{U}}{f_{1}^{U}-f_{1}^{L}}, \zeta=\frac{f_{1}^{U} f_{2}^{U}-f_{1}^{L} f_{2}^{L}}{f_{1}^{U}-f_{1}^{L}},
$$


and

$$
g_{\text {new }}^{c c}(z)=\max \left\{\begin{array}{l}
\min \left\{\begin{array}{l}
f_{2}^{L} f_{1}^{c v}(z)+f_{1}^{U} \operatorname{mid}\left(f_{2}^{c v}(z), f_{2}^{c c}(z), \kappa f_{1}^{c v}(z)+\zeta\right)-f_{1}^{U} f_{2}^{L}, \\
f_{2}^{U} f_{1}^{c v}(z)+f_{1}^{L} \operatorname{mid}\left(f_{2}^{c v}(z), f_{2}^{c c}(z), \kappa f_{1}^{c v}(z)+\zeta\right)-f_{1}^{L} f_{2}^{U}
\end{array}\right\}, \\
\min \left\{\begin{array}{l}
f_{2}^{L} f_{1}^{c c}(z)+f_{1}^{U} \operatorname{mid}\left(f_{2}^{c v}(z), f_{2}^{c c}(z), \kappa f_{1}^{c c}(z)+\zeta\right)-f_{1}^{U} f_{2}^{L}, \\
f_{2}^{U} f_{1}^{c c}(z)+f_{1}^{L} \operatorname{mid}\left(f_{2}^{c v}(z), f_{2}^{c c}(z), \kappa f_{1}^{c c}(z)+\zeta\right)-f_{1}^{L} f_{2}^{U}
\end{array}\right\}, \\
\min \left\{\begin{array}{l}
f_{2}^{L} \operatorname{mid}\left(f_{1}^{c v}(z), f_{1}^{c c}(z), \frac{f_{2}^{c v}(z)-\zeta}{\kappa}\right)+f_{1}^{U} f_{2}^{c v}(z)-f_{1}^{U} f_{2}^{L}, \\
f_{2}^{U} \operatorname{mid}\left(f_{1}^{c v}(z), f_{1}^{c c}(z), \frac{f_{2}^{c v}(z)-\zeta}{\kappa}\right)+f_{1}^{L} f_{2}^{c v}(z)-f_{1}^{L} f_{2}^{U}
\end{array}\right\}, \\
\min \left\{\begin{array}{l}
f_{2}^{L} \operatorname{mid}\left(f_{1}^{c v}(z), f_{1}^{c c}(z), \frac{f_{2}^{c c}(z)-\zeta}{\kappa}\right)+f_{1}^{U} f_{2}^{c c}(z)-f_{1}^{U} f_{2}^{L}, \\
f_{2}^{U} \operatorname{mid}\left(f_{1}^{c v}(z), f_{1}^{c c}(z), \frac{f_{2}^{c c}(z)-\zeta}{\kappa}\right)+f_{1}^{L} f_{2}^{c c}(z)-f_{1}^{L} f_{2}^{U}
\end{array}\right\}, \\
\min \left\{\begin{array}{l}
f_{2}^{L} f_{1}^{c v}(z)+f_{1}^{U} f_{2}^{c c}(z)-f_{1}^{U} f_{2}^{L}, \\
f_{2}^{U} f_{1}^{c v}(z)+f_{1}^{L} f_{2}^{c c}(z)-f_{1}^{L} f_{2}^{U}
\end{array}\right\}, \\
\min \left\{\begin{array}{l}
f_{2}^{L} f_{1}^{c c}(z)+f_{1}^{U} f_{2}^{c v}(z)-f_{1}^{U} f_{2}^{L}, \\
\left.f_{2}^{U} f_{1}^{c c}(z)+f_{1}^{L} f_{2}^{c v}(z)-f_{1}^{L} f_{2}^{U}\right\}
\end{array}\right\}
\end{array}\right\}
$$

with

$$
\kappa=\frac{f_{2}^{U}-f_{2}^{L}}{f_{1}^{U}-f_{1}^{L}}, \zeta=\frac{f_{1}^{U} f_{2}^{L}-f_{1}^{L} f_{2}^{U}}{f_{1}^{U}-f_{1}^{L}} .
$$

Lemma 1 shows that formulas (5) and (6) are correct.

Lemma 1 The closed-form solution for $g^{c v}(z)$ given by (1) is given by (5) and the closedform solution for $g^{c c}(\boldsymbol{z})$ given by (2) is given by (6).

Proof We prove validity of the convex formula (5). The proof for the validity of (6) is analogous.

Problem (1) minimizes $\max \left\{H_{1}(\boldsymbol{x}), H_{2}(\boldsymbol{x})\right\}$ over the two-dimensional box $\mathbb{B}=\left[f_{1}^{c v}(\boldsymbol{z}), f_{1}^{c c}(\boldsymbol{z})\right] \times\left[f_{2}^{c v}(\boldsymbol{z}), f_{2}^{c c}(\boldsymbol{z})\right]$. Let $\boldsymbol{x}^{*}$ denote an optimal solution point of problem (1), which by compactness exists. If it holds that $H_{1}\left(x^{*}\right) \neq H_{2}\left(x^{*}\right)$, then (1) is equivalent to minimizing the appropriate $H_{j}(\boldsymbol{x})$ over $\mathbb{B}$, where $j$ is determined by

$$
j \in \arg \max _{i \in\{1,2\}} H_{i}\left(\boldsymbol{x}^{*}\right) .
$$

With $j$ determined by (7), problem (1) reduces to a linear program. Indeed, there exists a neighborhood of $\boldsymbol{x}^{*}, \mathcal{N}\left(\boldsymbol{x}^{*}, \varepsilon\right)$, such that $H_{j}(\boldsymbol{x})=\max \left\{H_{1}(\boldsymbol{x}), H_{2}(\boldsymbol{x})\right\}$ for all $\boldsymbol{x} \in \mathcal{N}\left(\boldsymbol{x}^{*}, \varepsilon\right)$, yielding

$$
H_{j}\left(\boldsymbol{x}^{*}\right)=\min _{\boldsymbol{x} \in \mathbb{B} \cap \mathcal{N}\left(\boldsymbol{x}^{*}, \varepsilon\right)} H_{j}(\boldsymbol{x})=\min _{\boldsymbol{x} \in \mathbb{B}} H_{j}(\boldsymbol{x}),
$$

where the second equality follows from convexity of $H_{j}$.

Therefore, a solution of (1) has to lie at one of the corners of $\mathbb{B}$, given by the set $\mathbb{B}^{c}=\{D L, U L, D R, U R\}$, with

$$
\begin{aligned}
& D L=\left(f_{1}^{c v}(\boldsymbol{z}), f_{2}^{c v}(\boldsymbol{z})\right), U L=\left(f_{1}^{c v}(\boldsymbol{z}), f_{2}^{c c}(\boldsymbol{z})\right), \\
& D R=\left(f_{1}^{c c}(\boldsymbol{z}), f_{2}^{c v}(\boldsymbol{z})\right), U R=\left(f_{1}^{c c}(\boldsymbol{z}), f_{2}^{c c}(\boldsymbol{z})\right) .
\end{aligned}
$$



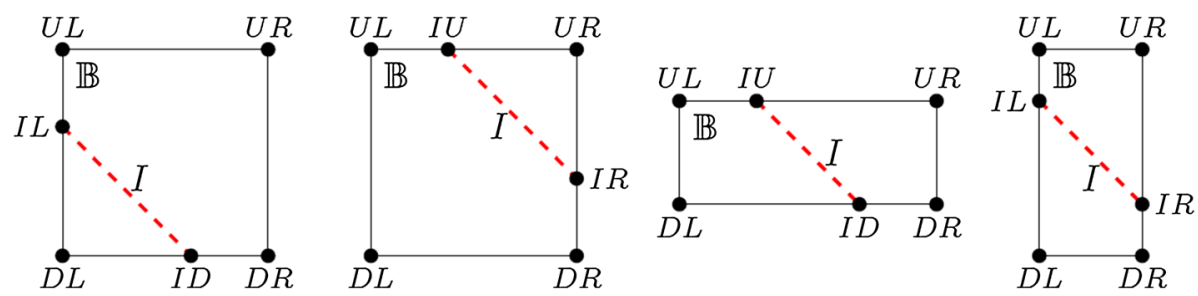

Fig. 3 Four possible cases of $\cap I \neq \emptyset$. The box $\mathbb{B}$ is given by the four corners $\{D L, U L, D R, U R\}$ and the two additional points emerge from the intersection with $I$. In each case the six corners are elements of $\hat{P}=\mathbb{B} \cap P . I$ is represented by the dashed line

If, on the contrary, $H_{1}\left(x^{*}\right)=H_{2}\left(x^{*}\right)$, it follows that the intersection of $\mathbb{B}$ with the line $I=\left\{\boldsymbol{x} \mid x_{2}=\kappa x_{1}+\zeta\right\}$, with $\kappa$ and $\zeta$ defined in (5), is non-empty and that (1) is equivalent to minimizing $H_{1}(\boldsymbol{x})$ over $\mathbb{B} \cap I$. Indeed,

$$
\min _{\boldsymbol{x} \in \mathbb{B} \cap I} H_{1}(\boldsymbol{x}) \geq \min _{\boldsymbol{x} \in \mathbb{B}} H_{1}(\boldsymbol{x}) \geq H_{1}\left(\boldsymbol{x}^{*}\right)
$$

and

$$
\min _{\boldsymbol{x} \in \mathbb{B} \cap I} H_{1}(\boldsymbol{x}) \leq \min _{\boldsymbol{x} \in \mathbb{B} \cap I} \max \left\{H_{1}(\boldsymbol{x}), H_{2}(\boldsymbol{x})\right\}=H_{1}\left(\boldsymbol{x}^{*}\right),
$$

yielding $\min _{\boldsymbol{x} \in \mathbb{B} \cap I} H_{1}(\boldsymbol{x})=H_{1}\left(\boldsymbol{x}^{*}\right) . \mathbb{B} \cap I$ is the non-empty intersection of a line with a box and is either a point or a line segment. Therefore, also in this case, (1) is equivalent to a linear program with an optimal solution at the edge of the (potentially degenerate) intersection $\mathbb{B} \cap I$. The intersection of $I$ with the lines defining the box $\mathbb{B}$, see Fig. 3, give the set $I^{c}=\{I L, I R, I D, I U\}$ of candidate points for an optimal solution, with

$$
\begin{aligned}
I L & =\left(f_{1}^{c v}(z), \kappa f_{1}^{c v}(z)+\zeta\right), I R=\left(f_{1}^{c c}(z), \kappa f_{1}^{c c}(z)+\zeta\right) \\
I D & =\left(\frac{f_{2}^{c v}(z)-\zeta}{\kappa}, f_{2}^{c v}(z)\right), I U=\left(\frac{f_{2}^{c c}(z)-\zeta}{\kappa}, f_{2}^{c c}(z)\right) .
\end{aligned}
$$

It follows that the union $P=\mathbb{B}^{c} \cup I^{c}$, always includes an optimal solution to problem (1), which can be reformulated as

$$
\min _{\boldsymbol{x} \in \mathbb{B} \cap P} \max \left\{H_{1}(\boldsymbol{x}), H_{2}(\boldsymbol{x})\right\} .
$$

By definition we have $\mathbb{B}^{c} \subset \mathbb{B}$. Furthermore, let $M\left(\alpha_{1}, \alpha_{2}, \alpha_{3}\right)$ with $\alpha_{i} \in \mathbb{R}^{2}$ be a mapping that maps three collinear points to the middle one, and let

$$
\begin{aligned}
& \widehat{I L}=M(D L, U L, I L), \widehat{I R}=M(D R, U R, I R), \\
& \widehat{I D}=M(D L, D R, I D), \widehat{I U}=M(U L, U R, I U) .
\end{aligned}
$$

Note that, although the domain of $M\left(\alpha_{1}, \alpha_{2}, \alpha_{3}\right)$ is $\mathbb{R}^{2}$, it can be expressed by the one dimensional mid (...) term, but we still introduce $M(\ldots)$ to avoid confusion. With $\hat{I}^{c}$ $=\{\widehat{I L}, \widehat{I R}, \widehat{I D}, \widehat{I U}\}$ and $\hat{P}=\mathbb{B}^{c} \cup \hat{I}^{c}$, it is easy to see that $\hat{P}=\mathbb{B} \cap P$. Observe, for example, that $\widehat{I L}=I L$ if and only if $I L \in \mathbb{B}$; otherwise, it evaluates to $D L$ or $U L$. Therefore (1) is further equivalent to

$$
\min _{\boldsymbol{x} \in \hat{P}} \max \left\{H_{1}(\boldsymbol{x}), H_{2}(\boldsymbol{x})\right\} .
$$


This is a closed-form solution. It remains to show that we can drop $U L$ and $D R$ from $\hat{P}$, obtaining the proposed corrected formula (5), without affecting the result.

We show it for the corner $U L$, the proof for $D R$ is analogous. We argue that $U L$ is given either by $M(U L, U R, I U)$ or $M(D L, U L, I L)$. Assume to the contrary that $I U$ is to the right of $U L$ and $I L$ is below $U L$. That is, assume that $\frac{f_{2}^{c c}(z)-\zeta}{\kappa}>f_{1}^{c v}(z)$ and $\kappa f_{1}^{c v}(z)+\zeta<f_{2}^{c c}(z)$. This would imply that $I U$ is to the right and above $I L$ and that the line $I$, passing from $I U$ to $I L$ has positive slope, contradicting $\kappa<0$.

Note that formula (3) in [1], in addition to $U L$ and $D R$, also incorrectly dropped $D L$ and $U R$.

Consider Example 1 again, with the correct closed form (5). We obtain the correct relaxation shown in Fig. 2.

\section{Reference}

1. Tsoukalas, A., Mitsos, A.: Multivariate McCormick relaxations. J. Global Optim. 59, 633-662 (2014) 